\title{
Design and Implementation of Wireless Temperature Controller Based on Zigbee
}

\author{
HAO Qinxia \\ School of Communication and Information Engineering, Xi'an University of Science and Technology, Xi'an 710054, China \\ haoqinxia@xust.edu.cn
}

\begin{abstract}
This paper introduces a design of wireless temperature controller hardware platform based on ZigBee. Wireless temperature controller using ZigBee to build intelligent household heating control system. By CC2530 chip consis the core and related peripheral circuit. Interworking user can realize the information through the mobile comsts of wireless temperature controller hardware platform amunication network, Internet and intelligent household heating control system, Real-time monitoring of heating information home.

Index Terms - ZigBee, UC/OS - II, CC2530, Wireless controller hardware platform
\end{abstract}

\section{Introduction}

During the winter heating period, most of the city in our country use the central heating, and 24 hours a day continuous heating. The heating system consider little about if there are people in the house, everyone has their own require for the room temperature, use cannot real-time ,mobile control the heating equipment, user passive using heating, cannot regulate the room temperature by themselves, heating energy consumption of huge. For saving efficiency, the Ministry of Construction provides residential heating progressive realization of household metering charges. While most of the the present household metering heating system use local control, cannot achieve the real-time, mobile collection information for the heating equipment. The monitoring command cannot mobile, real-time communicated to the heating equipment. Monitoring and control of the heating equipment can not achieve full-duplex mode.

In recent years, with the development of communication technology, wireless transmission of information is more and more stable and reliable, its anti-jamming capability, error correction capability and confidentiality growing ability, this makes real-time and mobile control the heating equipment become possible, heating equipment monitoring and control work in full-duplex mode. Based on the ZigBee network in the wireless personal area network, networking, and low - power sensor networks and other fields outstanding performance. The study presents a wireless ZigBee smart mobile controller system of household heating thermostat. The eventual realization users can use intelligent terminal control their own heating device status, achieve single-user control, just pay the actual using heating. Heating unit personnel use web access Heating Information Center query the heating, and achieve remote meter reading.

\section{II . Introduction of ZigBee Technology}

Zigbee is a wireless digital network short distance, low power consumption, high reliability. The ZigBee protocol from bottom to top, respectively, the physical layer (PHY), media access control (MAC) layer, transport layer, network layer (TL) (NWK), the application layer (APL), Provisions of the physical layer and media access control layer following the IEEE 802.15.4 standards. The main characteristics of ZigBee network is of low power consumption, low cost, low rate, support a large number of nodes, supports a variety of network topology, low complexity, fast, reliable, safe. Devices in ZigBee network can be divided into Coordinator (Coordinator), the sink node (Router), the sensor nodes (EndDevice) and other three kinds of roles, ZigBee network as shown in Figure 1.

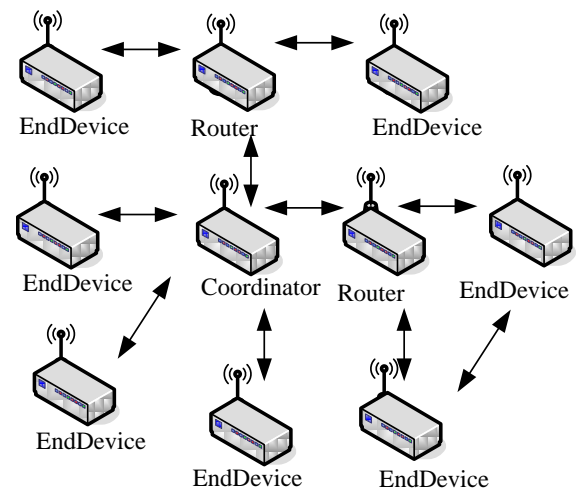

Fig.1 ZigBee networking mode

Zigbee network is a more than 65000 wireless data transmission module of a platform, a base station every Zigbee network digital modules like mobile network, within the scope of the entire network, they can communicate with each other, the distance between each node in the network can be from the standard 75 meters, to the expansion of the hundreds of meters, even a few kilometers. In addition to the entire Zigbee network can also be connected with various other existing network. The Zigbee network is mainly built for automatic control of data transmission, each Zigbee "base" is no more than 1000 yuan. Each Zigbee node in the network can not only be monitored objects, such as sensor is directly connected to data acquisition and monitoring, it can also automatically converted to other nodes of the network data transmission over 
data. In addition, each Zigbee node (FFD) but also in their coverage range, and a plurality of does not assume the network information transfer tasks of isolated nodes (RFD) wireless connection. Zigbee technology is targeting industrial, home automation, remote control, automation, agriculture automation and medical care, and it can also target such as the city of vehicle positioning of the local area mobile.

\section{III . System structure}

The smart household heating control system consists of the smart mobile terminal, heating Web information center, wireless thermostats and communication media section. The system structure of smart household heating control system is Figure 1.The data transmission divided into he the upper network and the underlying network, The underlying network use wireless temperature controller as the center, via the ZigBee star network, collect the information of the in and out temperature sensor and the temperature detecting sensor,after collect the data, using the coordinator send the data to the Web information center. The upper network use the Web information center as the center. After the data of center receive the wireless thermostats and analysis, put into the heating Web Information Center Database. Intelligent terminal based on Android through the $3 \mathrm{G}$ network access heating Web information center, the center real-time display the information from the underlying network controller. And pass the smart terminal control operation into a command to the wireless temperature controller, real-time control the heating flow of the heating equipment.

As we know through the above analysis, the wireless temperature controller play important effect on underlying data acquisition and control, Its main functions are as follows:Via the ZigBee network collecting the information of the in and out temperature sensor and the temperature detecting sensor.In the star network, via coordinator package the data in the ZigBee network and upload to the Web information center.Response and execute the command from user send by the mobile terminal and the Web client.Control every digital flow valve on the household heating equipment.

\section{The hardware structure of wireless temperature controller}

The Wireless temperature controller design is divided into data acquisition module, data control module, data processing module,data communication module and power supply module,CC2530 within the integrated RF transceiver, industry leading rich on-chip and robust DMA controller. he specified performance of RF transceiver is far superior to the requirements of IEEE802.15.4 standard. Rich on-chip, such as AES coprocessor, and SPI communication interface, for a variety of applications in ZigBee, 6LoWPAN and RF4CE based protocol provides powerful support. Robust DMA controller for CC2530 in high strength, high throughput and real-time requirements of excellence strictly and other fields to provide a reliable guarantee. The enhanced 8051CPU in an industry standard, in-system programmable flash memory, 8-
KB RAM provides a reliable guarantee for the data processing. Therefore, using CC2530 as the core of the whole hardware structure, It integrates the data transmission between the wireless controller of the data acquisition module, data control module and each sensor I/O resource, integrated data processing module, and data communication module. The hardware structure of wireless temperature controller as shown in figure 2.

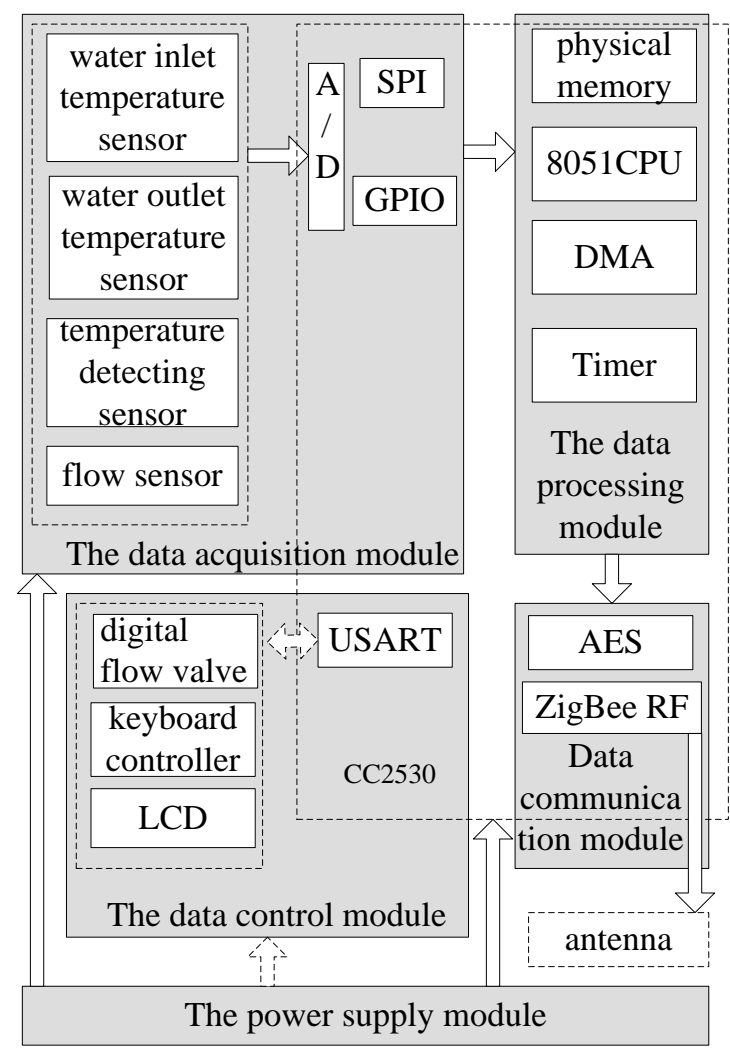

Fig.2 The hardware structure of wireless temperature controller

\section{A. The data acquisition module}

Data acquisition module includes a water inlet \& out temperature sensor, temperature detecting sensor, flow sensor and CC2530 A/D converter, serial communication interface. Among them, the water inlet temperature sensor is installed on the water inlet pipe, the out water temperature sensor is installed in the return pipe, the temperature detecting sensor mounted on a heat sink. When the digital flow valve is opened, the hot water with higher temperature into the heat exchanger or heat sink from the water inlet pipe, in the release of some heat, with lower the temperature outflow from return water pipe.At this time, the flow sensor generates a corresponding flow pulse signal data processing module, Take a multiplyaccumulate instruction to handle the receive temperature difference signal and traffic signal. The temperature sensor uses digital temperature sensor DS18B20 produced by Dallas semiconductor. The measurement of the temperature range of $55{ }^{\circ} \mathrm{C}-125{ }^{\circ} \mathrm{C}$, in range $-10{ }^{\circ} \mathrm{C}-85{ }^{\circ} \mathrm{C}$, accuracy is $\pm 0.5{ }^{\circ} \mathrm{C}$, fully meet the design of the temperature measurement range 
and accuracy requirements. Finish the data acquisition, using the $\mathrm{A} / \mathrm{D}$ converter is sent to the data processing module.

\section{$B$. The data processing module}

The data processing module is responsible for management and coordination of each hardware module, received from the signal acquisition module, signal into the backwater temperature difference $\Delta \mathrm{t}$ and traffic signal $\mathrm{V}$, and calculate the Q subroutine heat through a special instruction MAC and thermal calculation.CC2530 chip use 8051 CPU core that is a 8051 single cycle compatible kernel. It has three different memory access bus (SFR, DATA CODE/XDATA), single cycle access to SFR, DATA and SRAM. The CPU and DMA controller and the physical memory and all peripherals connected through the SFR bus. using XDATA storage space access memory, access to all physical memory. Each channel (trigger, priority, mode of transmission, addressing mode, the source and destination pointer and transmission count) using DMA descriptors configuration in any local memory. Many hardware peripherals (AES kernel, flash memory controller, USART, timer, ADC interface) by transmitting data between SFR or XREG address and flash memory/SRAM memory using the DMA controller, to achieve high efficiency operation.

\section{The data control module}

The data control module includes a digital flow valve, LCD and keyboard controller, and through the USART directly with the data processing module. Digital flow valve is a control liquid flow valve, installed in the water inlet pipe, consisted of the stepper motor, the mechanical conversion device and read door. Via Control pulse sequence to adjust the step angle and steering of stepping motor, in order to control valve, regulating the flow of water, and then change the indoor temperature.LCD is an information display unit, used to display the current indoor temperature and input temperature value, LCD using the FSTN display LCD module LM6059BCW model. The keyboard controller buttons including 0-9 digital key and temperature state key and input state key manually switch the temperature state, and send the temperature data to the data control center, through the ZigBee network transfer to the heating of the Web Information Center, so as to statistic the user heat metering.

\section{Data communication module}

CC2530 IEEE has a 802.15.4 compatible wireless transceiver, RF kernel control simulation module. ZigBee protocol data transmission procedure is the application layer data from one ZigBee device to another ZigBee device, it is a process that each layer to package and each layer to parse. In star network structure has information collection node and gateway node two kinds of equipment, there are two kinds of data, two interactive data: environmental information data and user command data. Information collection node transmit environment information data to the gateway node, the gateway node communication user command to the information collection node. So the system through the ZigBee $\mathrm{RF}$ realized full duplex communication.

\section{E. The power supply module}

Because CC5230 for digital core and peripherals provides a $1.8-\mathrm{V}$ low dropout voltage regulator power supply. It provides power management feature, could achieve long battery life using different power supply mode low power operation. Wireless temperature controller also need to provide a stable voltage to CC5230 hardware, design using UPS and TPS73HD318 power module to provide uninterrupted power supply.

\section{The software structure of wireless temperature controller}

\section{A. Software module design}

Wireless temperature control use modular programming ideas, according the function division module and design, through C/OS - II embedded real-time scheduling multitask operating system each function module of the task orderly work. Based on real-time operating system above are developed by $\mathrm{C}$ programming language the initialization procedure, temperature acquisition program, flow acquisition program, digital flow valve switching, heat calculation program, LCD driver, keyboard scanning procedures, serial communication driver program and ZigBee communication module, and divide the task according to the prioritization. The software structure of the system is shown in Figure 3.Using this software can realize the user control the heating equipment by the state of the mobile terminal, and calculate the corresponding heating expense according to the actual heat consumption. The heating unit staff queries users heating information through the access check heating Web Information Center, remote meter reading.

\begin{tabular}{|c|c|c|}
\hline \multirow{3}{*}{} & \multicolumn{3}{|c|}{ Function } \\
\cline { 2 - 3 } & Temperature acquisition & Heat calculation \\
\cline { 2 - 3 } & Traffic data collection & LCD driver \\
\cline { 2 - 3 } & Serial communication driver & Key scan \\
\cline { 2 - 3 } & Digital flow valve switch & ZigBee driver \\
\cline { 2 - 3 } & \multicolumn{3}{|c|}{ CC2530 } \\
\hline USRT & \multicolumn{2}{|c|}{ serial port1 } \\
\hline GPIO & \multicolumn{2}{|c|}{ serial port2 } \\
\hline SPI & \multicolumn{2}{|c|}{ serial port3 } \\
\hline
\end{tabular}

Fig3 The software structure of the system

\section{B. The data flow design}

According to the function, the wireless controller task is mainly divided into 5 categories: timing data acquisition, data processing tasks, monitoring task, task communication and human-computer interaction task. Its Data processing is: timing acquisition task, trigger timing by Timers, network collection into the backwater temperature sensor, temperature sensor and flow sensor data, ata processing tasks, classification processing acquisition task of received data, updating data and 
save to physical memory, the monitoring task, according to the mobile terminal command regulation of digital flow valve working condition, in the LCD display temperature data, the communication task, through the ZigBee network, collecting and processing data in time and response for every command the user send. Human-computer interaction tasks, wireless thermostat providing interactive visualization, according to the keyboard input to view the running state of the system, historical data and configuration of some of the basic operation parameters of the system.

\section{VI . Conclusion}

This text gives a solution about intelligent household heating control system, the scheme not only can real-time and remotely collect data information of every household heating, and the mobile terminal user temperature control command could execution timely in the user's heating equipment, realize full-duplex mode monitoring and control, timely, and effectively avoid energy waste, according to the actual demand of user with the least amount of heat to achieve the best effect of heating. Through the wireless controller ZigBee network, problems of energy waste and inadequate heating etc appeared today, with its novel design and good application prospect, big use value , It must be widely used.

\section{References}

[1] Wu Renjie. Based on ZigBee Intelligent Household Saving Energy Control System Design. Computer Measurement \& Control, 2012.20(7): 1818-1820.

[2] YANG Tao, WANG Bo, WANG Ping,DU Zongwei. Design of Wireless Security Monitoring System Based on ZigBee and ARM. Agriculture Network Information.2011.(11):26-29.

[3] DAI Guang - xian. Study of Mine Shaft Personnel Positioning System Based on ZigBee Technology. Coal Technology. 2012.1(31):106-106.

[4] ZHANG Yongqiang, XIAO Jun,FU Qiufeng. An applied reaserch of heating meter and intelligent temperature control based on ARM. Industrial Instrumentation \& Automation, 2010 (1):55-56.

[5] Yang Linnan, Gao Lutao, Lin Ersheng. Intelligent diagnose system of diseases and insect pests in sweet corn based on mobile terminal with Android system. Transactions of the Chinese Society of Agricultural Engineering, 201.(9):163-164.

[6] Li Changfeng Teng Guoku Chang Chuang. Design of Wireless Sensor Networks of Gateways Based on ZigBee. Computer \& Digital Engineering, 2011.7 (39):150-153.

[7] Song Xiaoqian, Zhou Dongsheng. Development and Researh of Application Based on Android Platform. Software Guide,2011.10(2): 104-106.

[8] Wang Ligang, Mu Haiwei, Liu Shiqing. Research and Design of Embedded Indoor Intelligent Temperature Controller. Journal of Northeast Forestry University.2009.(6):64-65 